\title{
EDITORIAL
}

\section{Multiplying publications}

One of the recurring topics of concern for the editors of scientific journals is the issue of multiple publication, where the same, or essentially the same, paper is published in two or more journals.

One hears frequent complaints from research scientists about the difficulty of keeping up with the literature, and multiple publication of the same work at its very least makes this even more difficult. At its worst it should be viewed as a kind of scientific fraud whereby the authors are attempting to expand their apparent contribution to knowledge and increase their numbers of publications in a way that deceives their peers. In publishing the same material twice such authors are breaching the spirit and possibly the legal aspects of copyright and, for many journals, breaking their written undertaking to the editor when submitting the paper for publication.

Our Directions to Contributors contain the wording 'Submission of a paper... will be held to imply that it (the paper)... not previously published; that is not under consideration for publication elsewhere...'. These words to me have the force of an undertaking that the work is original, that it is substantially different from any other paper that the authors have published, and that it is not being submitted to another journal simultaneously; in other words the British Journal of Nutrition has the first option on publishing the paper. We depend on the integrity of our peers in describing the results of their work and in their involvement in the review process, and multiple publication serves to erode that trust. I think that we may have to ask authors to state explicitly, in the letter accompanying the paper, that their paper is not being submitted to another journal.

I accept that one could see my remarks so far as those of an editor who feels that he has been let down and it set me wondering about the devil's advocate's position. Why should authors not be permitted to send their papers simultaneously to a range of suitable journals and see which one was able to deal with it most expeditiously? It would be analogous to sending out for tender and accepting the most favourable offer. In the case of a paper this would possibly mean minimal changes (no tiresome referee's report, no need to argue your way point by point with the editor) and rapid publication, possibly with typographical errors or worse, because the primary emphasis would be on speed of acceptance and publication as each journal's editor competed to get the paper into press first. The scenario would, I suspect, be very different: acceptance would be more arbitrary and at the whim of a narrow editorial group.

Such an approach would destroy scientific journals because so much editorial time and money would be wasted in refereeing and technical editing. Editorial teams and referees would act in the knowledge that much of their effort would be pointless because they would be beaten to the press.

If we accept multiple submission and publication, copyright protection will be severely eroded and the scientific literature will collapse. Multiple publication of the same paper is fortunately rare, but it exists in more subtle forms which, I believe, are equally damaging to scientific research. It arises from the difficulty of making the distinctions between papers that describe similar work but that are not identical. The order of the authors may be 
permuted or some parts of the protocol may differ and different sets of results presented, so the one piece of work is used to generate several publications.

I recognize that some journals set page lengths and so encourage authors to slice up their work into publishable chunks, and the continuous emphasis from managers and administrators on numbers of publications is a strong incentive to publish several papers, whereas if one looked at the material scientifically then one paper would make a greater contribution to knowledge. I am not sure how 'impact factors' would respond to such strategy but I know that, as a reader, a rounded paper that presents a piece of experimental work as a whole is preferable. This is one reason why we at the British Journal of Nutrition do not set page limits on papers. Often we suggest combining papers to make a stronger paper or to bring a relatively slight paper into a stronger one. This incidentally can save pages overall because we do not need to print two or more bibliographies that have many references in common.

Recently we were faced with a different type of multiple publication where an author had published in his native language and wanted to publish an English translation in the hope, one assumes, that the paper would be more widely read. In this case we declined the paper, but on reflection I think that we were too rigid in our approach. It is an unfortunate fact of life that publications in languages other than English may not receive the attention they deserve. This is a reflection on the limited language skills of many scientists. I think that it is very disappointing if an author cannot publish in his or her native language, and that by accepting translations we may be able to lead readers into these native language journals and the potential wealth of material there. We have to deal with the formal issues of copyright but with good will this is not a real obstacle.

When authors, in submitting papers to us, give us the first and exclusive option for assessing and publishing their work they place an obligation on the journal and the editorial team to act in the best interests of the author in dealing quickly and fairly with papers, and the obligations, as with all agreements, apply to both sides.

D. A. T. Southgate 